\title{
Doing Spin Physics with Unpolarized Particles
}

\author{
Igor P. Ivanov $\oplus^{*}$ \\ CFTP, Instituto Superior Tecnico, Universidade de Lisboa, Lisbon 1049-001, Portugal \\ Nikolai Korchagin $\oplus^{\dagger}$ \\ Institute of Modern Physics, Chinese Academy of Sciences, Lanzhou 730000, China \\ Alexandr Pimikov $\oplus^{*}$ \\ Institute of Modern Physics, Chinese Academy of Sciences, Lanzhou 730000, China \\ and Research Institute of Physics, Southern Federal University, Rostov-na-Donu 344090, Russia \\ Pengming Zhang $\oplus^{\S}$ \\ School of Physics and Astronomy, Sun Yat-sen University, Zhuhai 519082, China
}

(Received 4 December 2019; accepted 8 April 2020; published 13 May 2020)

\begin{abstract}
Twisted, or vortex, particles refer to freely propagating non-plane-wave states with helicoidal wave fronts. In this state, the particle possesses a nonzero orbital angular momentum with respect to its average propagation direction. Twisted photons and electrons have been experimentally demonstrated, and creation of other particles in twisted states can be anticipated. If brought in collisions, twisted states offer a new degree of freedom to particle physics, and it is timely to analyze what new insights may follow. Here, we theoretically investigate resonance production in twisted photon collisions and twisted $e^{+} e^{-}$annihilation and show that these processes emerge as a completely novel probe of spin and parity-sensitive observables in fully inclusive cross sections with unpolarized initial particles. This is possible because the initial state with a nonzero angular momentum explicitly breaks the left-right symmetry even when averaging over helicities. In particular, we show how one can produce almost $100 \%$ polarized vector mesons in unpolarized twisted $e^{+} e^{-}$annihilation and how to control its polarization state.
\end{abstract}

DOI: 10.1103/PhysRevLett.124.192001

Introduction.-Spin-parity properties of hadrons are a fascinating chapter in modern particle phenomenology. The rich hadron spectrum exhibits a variety of spin-parity quantum numbers possible in the $q \bar{q}$ and $q q q$ quark combinations and in multiquark states [1]. Deep inelastic scattering (DIS) with a polarized lepton or proton allows one to investigate how spin of the ultrarelativistic proton emerges from spins and orbital angular momenta of its constituents [2-4]. Reconstructing the proton spin structure in $3 \mathrm{D}$ brings in new spin-sensitive variables, which can be encoded via transverse momentum distributions and explored experimentally in semi-inclusive DIS with transversely polarized protons [5].

The spin-parity structure of hadrons is explored in experiment via two approaches: either one collides polarized initial particles and investigates the response of the cross section to flipping the polarization sign or direction,

Published by the American Physical Society under the terms of the Creative Commons Attribution 4.0 International license. Further distribution of this work must maintain attribution to the author(s) and the published article's title, journal citation, and DOI. Funded by SCOAP . or one studies exclusive or semi-inclusive reactions and reconstructs the spin properties of the target hadron or intermediate resonances from the final state angular distributions. There seems to be no other way to access spindependent observables.

Here, we propose a novel tool for spin physics in particle collisions, complementary to all existing approaches. We demonstrate that parity- and spin-sensitive observables can be accessed in fully inclusive processes with unpolarized initial particles-provided they are prepared in the so-called twisted states. Whether this method, with all technical details taken into account, is superior over the traditional approaches in specific processes requires detailed dedicate analyses. The goal of this Letter is to demonstrate that this new tool exists and that it allows one to probe particles in a way that would be unthinkable in the usual plane wave scattering. For this purpose, we calculate the simplest process $2 \rightarrow 1$, a resonance production in collision of two twisted particles, and show how new spin and parity-sensitive observables emerge in it.

Twisted photons and electrons.-A twisted particle, be it a photon, an electron, or a hadron, is a wave packet with helicoidal wave fronts. It propagates, as a whole, in a 
certain direction and carries a nonzero orbital angular momentum (OAM) projection with respect to that direction. Twisted photons have been known since long ago [6-9]; following suggestions of [10], twisted electrons $[11,12]$ were also recently created [13-15]. Unlike spin, the OAM can reach arbitrarily high values; twisted electrons with OAM as large as 100 have been demonstrated experimentally. Relying on ideas of how to bring them into the GeV energy range $[16,17]$ and steer them in accelerators [18], and on future experimental progress, one can imagine collider-type experiments with GeV-range twisted photons or twisted $e^{+} e^{-}$annihilation.

Description of twisted particles adapted to calculation of their high energy collisions was presented in $[16,17]$ and further developed in [19-21]; see also recent reviews $[9,11]$ and the Supplemental Material [22]. For each sort of field (photons, electrons, etc.), one begins with Bessel twisted states $|E, \varkappa, m\rangle$, which represent solutions of the free wave equation with definite energy $E$, longitudinal momentum $k_{z}$, modulus of the transverse momentum $\left|\mathbf{k}_{\perp}\right|=\varkappa$, the $z$ projection of the total angular momentum (AM) $m$, and definite helicity. (Natural units $\hbar=c=1$ are used; 3D vectors are given by bold symbols, and the transverse momenta are labeled with the subscript $\perp$.) A Bessel twisted photon is defined, in the Coulomb gauge, as

$$
\mathbf{A}_{\varkappa m k_{z} \lambda}(\mathbf{r})=e^{i k_{z} z} \int a_{\varkappa m}\left(\mathbf{k}_{\perp}\right) \mathbf{e}_{\mathbf{k} \lambda} e^{i \mathbf{k}_{\perp} \mathbf{r}_{\perp}} \frac{d^{2} \mathbf{k}_{\perp}}{(2 \pi)^{2}},
$$

where the Fourier amplitude $a_{\varkappa m}\left(\mathbf{k}_{\perp}\right)$ is given by

$$
a_{\varkappa m}\left(\mathbf{k}_{\perp}\right)=i^{-m} e^{i m \varphi_{k}} \sqrt{\frac{2 \pi}{\varkappa}} \delta\left(\left|\mathbf{k}_{\perp}\right|-\varkappa\right) .
$$

It is a superposition of plane wave (PW) photons with equal $E, k_{z},\left|\mathbf{k}_{\perp}\right|=k \sin \theta$, and helicity $\lambda= \pm 1$, but arriving from different azimuthal angles $\varphi_{k}$. Each PW component of a twisted photon contains its polarization vector $\mathbf{e}_{\mathbf{k} \lambda}$, orthogonal to its momentum: $\mathbf{e}_{\mathbf{k} \lambda} \mathbf{k}=0$. Notice that a twisted photon with given total AM $m$ and helicity $\lambda$ is not an eigenstate of the OAM $z$-component operator $\hat{L}_{z}=-i \partial / \partial \varphi_{k}$ nor of the spin $z$-component operator $\hat{s}_{z}$. This is a manifestation of the spin-orbital interaction of light, which gives rise to a variety of remarkable optical phenomena [31]. Nevertheless, in the paraxial approximation $x /\left|k_{z}\right|=|\tan \theta| \ll 1$, the spin-orbital coupling is suppressed and one can deal with approximately conserved $s_{z} \approx \lambda$ and $L_{z} \approx \ell \equiv m-\lambda$.

A Bessel twisted electron $[11,21,32]$ is a monochromatic solution of the Dirac equation with definite $E, k_{z}, \varkappa$, halfinteger total AM $m$, and helicity $\zeta= \pm 1 / 2$,

$$
\Psi_{\varkappa m k_{z} \zeta}(\mathbf{r})=e^{i k_{z} z} \int a_{\varkappa m}\left(\mathbf{k}_{\perp}\right) \frac{u_{\zeta}(k)}{\sqrt{2 E}} e^{i \mathbf{k}_{\perp} \mathbf{r}_{\perp}} \frac{d^{2} \mathbf{k}_{\perp}}{(2 \pi)^{2}},
$$

with the standard expressions for the plane wave bispinor $u_{\zeta}(k)$ and with the same Fourier amplitude $a_{\varkappa m}\left(\mathbf{k}_{\perp}\right)$ as in Eq. (2). A similar expression holds for the negative frequency solutions of the Dirac equation $v_{\zeta}(k)$ used to describe positrons. Again, spin and OAM $z$ projections are not separately conserved in twisted electrons due to the intrinsic spin-orbital interaction [32,33], but in the paraxial approximation both $s_{z} \approx \zeta$ and $\ell=m-\zeta$ are approximately conserved.

A pure Bessel state $|E, \varkappa, m\rangle$ is not normalizable in the transverse plane. We construct a realistic normalizable monochromatic twisted beam as a superposition of Bessel states with equal $E, m$, and helicity but with a distribution over $\varkappa,|E, \bar{\varkappa}, \sigma, m\rangle=\int d \varkappa f(\varkappa)|E, \varkappa, m\rangle$, with a weight function $f(\varkappa)$ peaked at $\bar{\varkappa}$ and having a width $\sigma$.

Some care is needed to define what an unpolarized twisted photon or electron beam is. For PW initial particles, the polarization and coordinate degrees of freedom factorize, and the definition of the unpolarized cross section is straightforward. For twisted photons and electrons, they are coupled, so that there is no unique definition of unpolarized twisted photon or electron beam. In our calculations, we define an unpolarized twisted beam as an equal mixture of the two helicity configurations calculated for the same value of $m$. Alternative definitions as well as the sensitivity of the results will be treated at length in a detailed follow-up paper [34]. We only stress here that the effects discussed below are present for any definition.

Resonance production in twisted particle collisions.We apply the formalism developed in $[16,20]$ to a generic $2 \rightarrow 1$ process of resonance production in annihilation of two counterpropagating twisted initial particles defined with respect to the same axis $z$ with energies $E_{1}, E_{2}$, moduli of the transverse momenta $\varkappa_{1}, \varkappa_{2}$, and AM $m_{1}$ and $m_{2}$, respectively. For simplicity, the two initial particles are assumed to be massless. The final particle with mass $M$ is described in terms of plane waves with momentum $\mathbf{K}$ and energy $E_{K}$.

The $S$-matrix amplitude of twisted particle annihilation can be written as a superposition of PW $S$ amplitudes $S_{\mathrm{PW}}$ with different transverse momenta of the initial particles,

$$
\begin{aligned}
S & =\int \frac{d^{2} \mathbf{k}_{1 \perp}}{(2 \pi)^{2}} \frac{d^{2} \mathbf{k}_{2 \perp}}{(2 \pi)^{2}} a_{\varkappa_{1} m_{1}}\left(\mathbf{k}_{1 \perp}\right) a_{\varkappa_{2},-m_{2}}\left(\mathbf{k}_{2 \perp}\right) S_{\mathrm{PW}} \\
& =i(2 \pi)^{4} \frac{\delta(\Sigma E) \delta\left(\Sigma k_{z}\right)}{\sqrt{8 E_{1} E_{2} E_{K}}} \frac{(-i)^{m_{1}-m_{2}}}{(2 \pi)^{3} \sqrt{\varkappa_{1} \varkappa_{2}}} \mathcal{J}
\end{aligned}
$$

where $\quad \delta(\Sigma E) \equiv \delta\left(E_{1}+E_{2}-E_{K}\right) \quad$ and $\quad \delta\left(\Sigma k_{z}\right) \equiv$ $\delta\left(k_{1 z}+k_{2 z}-K_{z}\right)$. The twisted amplitude $\mathcal{J}$ is defined as

$$
\begin{aligned}
\mathcal{J}= & \varkappa_{1} \varkappa_{2} \int d \varphi_{1} d \varphi_{2} e^{i m_{1} \varphi_{1}-i m_{2} \varphi_{2}} \\
& \times \delta^{(2)}\left(\mathbf{k}_{1 \perp}+\mathbf{k}_{2 \perp}-\mathbf{K}_{\perp}\right) \cdot \mathcal{M}
\end{aligned}
$$


where $\mathcal{M}$ is the usual PW invariant amplitude. The twisted amplitude $\mathcal{J}$ is nonzero only if $\varkappa_{i}=\left|\mathbf{k}_{i \perp}\right|$ and $K \equiv\left|\mathbf{K}_{\perp}\right|$ satisfy the triangle inequalities

$$
\left|\varkappa_{1}-\varkappa_{2}\right| \leq K \leq \varkappa_{1}+\varkappa_{2}
$$

and form a triangle with the area $\Delta$. Out of the many PW components "stored" in the initial twisted particles, the integral (5) receives contributions from exactly two plane wave combinations shown in Fig. 1: Combination (a) $\varphi_{1}=\varphi_{K}+\delta_{1}, \quad \varphi_{2}=\varphi_{K}-\delta_{2}, \quad$ and combination (b) $\varphi_{1}=\varphi_{K}-\delta_{1}, \varphi_{2}=\varphi_{K}+\delta_{2}$, where $\delta_{1}$ and $\delta_{2}$ are the inner angles of the triangle. As a result, $\mathcal{J}$ can be calculated exactly [19]

$$
\mathcal{J} \propto \frac{\varkappa_{1} \varkappa_{2}}{2 \Delta}\left[\mathcal{M}_{a} e^{i\left(m_{1} \delta_{1}+m_{2} \delta_{2}\right)}+\mathcal{M}_{b} e^{-i\left(m_{1} \delta_{1}+m_{2} \delta_{2}\right)}\right] .
$$

One observes the hallmark feature of twisted particle collisions: interference between two PW amplitudes $\mathcal{M}_{a}$ and $\mathcal{M}_{b}$ calculated for the two distinct initial PW pairs shown in Fig. 1 but the same final momentum $\mathbf{K}_{\perp}$.

The cross section can be written as

$$
d \sigma \propto|\mathcal{J}|^{2} \delta\left(E_{1}+E_{2}-E_{K}\right) d^{2} \mathbf{K}_{\perp} .
$$

The prefactor here is inessential since the new effects come not from the overall magnitude of the cross section but from its kinematic and helicity dependence. Integration with respect to $K$ can be used to eliminate the energy delta function in (8). For fixed $E_{i}, \varkappa_{i}$, and $M$, the energymomentum conservation fixes $K_{z}=k_{1 z}+k_{2 z}$ and, therefore, $K=\sqrt{E_{K}^{2}-M^{2}-K_{z}^{2}}$. Thus, the polar angle of the produced resonance is defined by

$$
\cos \theta_{K}=K_{z} / \sqrt{\left(E_{1}+E_{2}\right)^{2}-M^{2}} .
$$

The experiment can be repeated at different total energy $E_{K}=E_{1}+E_{2}$, but, as long as $E_{K}$ satisfies

$$
E_{-} \leq E_{K} \leq E_{+}, \quad E_{ \pm}=\sqrt{\left(\varkappa_{1} \pm \varkappa_{2}\right)^{2}+K_{z}^{2}+M^{2}},
$$
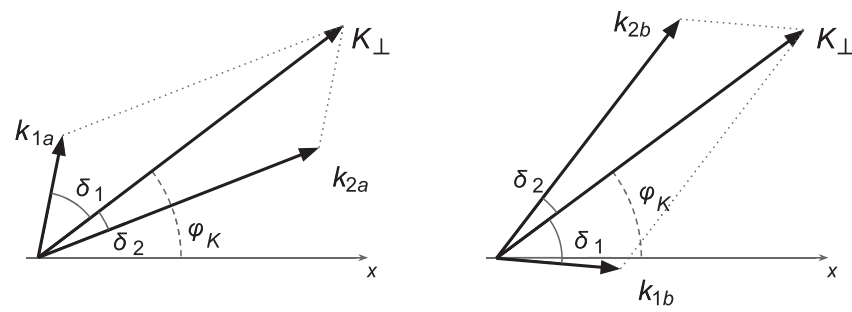

FIG. 1. The two kinematic configurations in the transverse plane that satisfy momentum conservation laws in scattering of two Bessel states. the resonance with mass $M$ can be produced with a nonzero cross section. The polar angle $\theta_{K}$ can also be adjusted by varying the ratio between $E_{1}$ and $E_{2}$.

Notice how different this situation is with respect to the PW collisions. In the PW case, the (narrow) resonance production cross section is $\propto \delta\left(E_{1}+E_{2}-E_{K}\right)$, and the production occurs only at the resonance. In twisted particle annihilation, there is a finite range of energies to produce a resonance with mass $M$. The cross section $\sigma\left(E_{K}\right)$ varies with $E_{K}$ in a periodic fashion, revealing the interference fringes induced by the varying coefficients in front of $\mathcal{M}_{a}$ and $\mathcal{M}_{b}$ in (7). Moreover, a twisted annihilation experiment running at fixed energy can simultaneously produce two or more resonances with close but different masses, provided they satisfy (10). According to (9), these resonances will be emitted at different polar angles. Thus, twisted annihilation has a built-in mass spectrometric feature [35].

Twisted particle collisions as parity analyzer.-To illustrate physics opportunities offered by twisted annihilation, consider production of a hypothetical spin-0 resonance in collision of two twisted photons [36]. It can be either a pure scalar $S$, a pure pseudoscalar $P$, or their mixture. For a pure scalar, whose effective interaction Lagrangian with photons is described by $\mathcal{L}_{S}=g F^{\mu \nu} F_{\mu \nu} S / 4$, one finds that the PW helicity amplitude is nonzero only for $\lambda_{1}=\lambda_{2}=\lambda$ : $\mathcal{M}_{S}=-2 g E_{1} E_{2} \delta_{\lambda_{1}, \lambda_{2}} \mathbf{e}_{1 \lambda} \mathbf{e}_{2 \lambda}$. For Bessel photons, one obtains the twisted amplitude $\mathcal{J}_{S} \propto \mathcal{J}_{1}+\lambda \mathcal{J}_{2}$ with

$\mathcal{J}_{1}=\cos \left(m_{1} \delta_{1}+m_{2} \delta_{2}\right)\left[\cos \left(\delta_{1}+\delta_{2}\right)\left(1-c_{1} c_{2}\right)-s_{1} s_{2}\right]$,

$\mathcal{J}_{2}=\sin \left(m_{1} \delta_{1}+m_{2} \delta_{2}\right) \sin \left(\delta_{1}+\delta_{2}\right)\left(c_{1}-c_{2}\right)$,

where we used the short notation $c_{i} \equiv \cos \theta_{i}$ and $s_{i} \equiv \sin \theta_{i}$. The cross section explicitly depends on the photon helicities: $\sigma_{\lambda}=\sigma_{0}+\lambda \sigma_{a}$, where $\sigma_{0} \propto \mathcal{J}_{1}^{2}+\mathcal{J}_{2}^{2}$ is the unpolarized cross section and $\sigma_{a} \propto 2 \mathcal{J}_{1} \mathcal{J}_{2}$ is the spin asymmetry.

This dependence on $\lambda$ may look surprising since the fundamental interaction is parity invariant. However, unlike in the PW case, here we explicitly break the left-right symmetry of the initial state. If the AM values $m_{i}$ are fixed, then the helicity choices $\lambda_{i}= \pm 1$ are not equivalent because the corresponding OAM contributions differ. The process would be invariant under the simultaneous sign flips $m_{i} \rightarrow-m_{i}$ and $\lambda_{i} \rightarrow-\lambda_{i}$, but not with respect to $\lambda_{i} \rightarrow-\lambda_{i}$ alone.

For a pure pseudoscalar with the interaction Lagrangian $\mathcal{L}_{P}=i g \epsilon_{\mu \nu \rho \sigma} F^{\mu \nu} F^{\rho \sigma} P / 8$, one gets $\mathcal{M}_{P}=\lambda \mathcal{M}_{S}$. If the spin-0 particle does not possess definite parity, one can write its $\mathrm{PW}$ production amplitude as $\mathcal{M}=a \mathcal{M}_{S}+b \mathcal{M}_{P}=$ $(a+\lambda b) \mathcal{M}_{S}$, where the (complex) coefficients $a$ and $b$ describe the scalar and pseudoscalar contributions to the amplitude. In the usual PW collision with circularly polarized photons, the cross section is $\sigma_{\lambda} \propto|a|^{2}+|b|^{2}+$ $2 \lambda \operatorname{Re}\left(a^{*} b\right)$. Averaging it over the initial photon polarizations 
yields $\sigma_{0} \propto|a|^{2}+|b|^{2}$; it reveals the overall production intensity but cannot measure the amount of scalarpseudoscalar mixing. Twisted photons offer access to this mixing even in the unpolarized cross section. Using the fixed- $m$ convention for unpolarized twisted photon beams, we obtain the twisted amplitude

$$
\mathcal{J}=\left(a \mathcal{J}_{1}+b \mathcal{J}_{2}\right)+\lambda\left(b \mathcal{J}_{1}+a \mathcal{J}_{2}\right),
$$

with $\mathcal{J}_{1}$ and $\mathcal{J}_{2}$ given in (11). Averaging $|\mathcal{J}|^{2}$ over the initial photon helicities, we get the unpolarized cross section

$$
\sigma_{0} \propto\left(\mathcal{J}_{1}^{2}+\mathcal{J}_{2}^{2}\right)\left(|a|^{2}+|b|^{2}\right)+4 \mathcal{J}_{1} \mathcal{J}_{2} \operatorname{Re}\left(a^{*} b\right) .
$$

Now both the total intensity $|a|^{2}+|b|^{2}$ and the scalarpseudoscalar mixing $\operatorname{Re}\left(a^{*} b\right)$ can be extracted experimentally from the energy dependence of $\sigma_{0}\left(E_{K}\right)$ and, specifically, from the exact location and heights of the interference fringes. Since $\mathcal{J}_{1}$ and $\mathcal{J}_{2}$ have different dependence on $\delta_{1}$ and $\delta_{2}$, their combinations $\mathcal{J}_{1} \mathcal{J}_{2}$ and $\mathcal{J}_{1}^{2}+\mathcal{J}_{2}^{2}$ produce different interference patterns and can be distinguished. Notice that, in contrast to polarized PW photon collisions, one does not need to flip the sign of the OAM and repeat the experiment to extract the parity-violating contribution. All relevant quantities can be extracted in a single run. A detailed numerical investigation of this effect with realistic twisted beams is delegated to Ref. [34].

Twisted annihilation as spin polarizer.-Our second example is production of a spin-1 resonance $V$ of mass $M$ in twisted $e^{+} e^{-}$annihilation. We take the PW helicity amplitude in the form

$$
\mathcal{M}_{\zeta_{1} \zeta_{2} \lambda_{V}}=g \bar{v}_{\zeta_{2}}\left(k_{2}\right) \gamma_{\mu} u_{\zeta_{1}}\left(k_{1}\right) V_{\lambda_{V}}^{\mu *}(K)
$$

where $\zeta_{1}, \zeta_{2}$, and $\lambda_{V}$ are the helicities of the electron, positron, and the produced vector resonance, respectively, and $V_{\lambda_{V}}^{\mu}$ is the polarization vector of the spin-1 resonance. To illustrate the effect, we evaluate this amplitude in the paraxial limit $\theta_{1} \rightarrow 0, \theta_{2} \rightarrow \pi$ but with generic $\theta_{K}$. It is nonzero only for $\zeta_{1}=-\zeta_{2} \equiv \zeta= \pm 1 / 2$,

$$
\mathcal{M}_{\zeta,-\zeta, \lambda_{V}} \propto e^{-i \zeta\left(\varphi_{2}+\varphi_{1}-2 \varphi_{K}\right)} \cdot\left(\lambda_{V} \cos \theta_{K}+2 \zeta\right) .
$$

For twisted $e^{+} e^{-}$annihilation, we get

$\mathcal{J}_{\zeta,-\zeta, \lambda_{V}} \propto\left(\lambda_{V} \cos \theta_{K}+2 \zeta\right) \cos \left[m_{1} \delta_{1}+m_{2} \delta_{2}-\zeta\left(\delta_{1}-\delta_{2}\right)\right]$,

where $m_{1}$ and $m_{2}$ are half-integers. The unpolarized twisted $e^{+} e^{-}$cross section is then

$$
\begin{aligned}
\sigma_{\lambda_{V}= \pm 1} \propto & \left(1+\cos ^{2} \theta_{K}\right) \\
& \times\left[1+\cos \left(2 m_{1} \delta_{1}+2 m_{2} \delta_{2}\right) \cos \left(\delta_{1}-\delta_{2}\right)\right] \\
& +2 \lambda_{V} \cos \theta_{K} \sin \left(2 m_{1} \delta_{1}+2 m_{2} \delta_{2}\right) \sin \left(\delta_{1}-\delta_{2}\right) .
\end{aligned}
$$

This result shows another remarkable phenomenon, which was impossible in the PW case. Even with unpolarized electrons and positrons, one produces unequal amounts of $\lambda_{V}=+1$ and $\lambda_{V}=-1$ vector mesons. The imbalance is enhanced at $\theta_{K}$ far from $\pi / 2$ and is sensitive to the exact position at the interference fringes. Thus, the produced vector meson is polarized on average and its polarization can be controlled.

To give a numerical example, we consider production of the $J / \psi$ meson with $M=3.1 \mathrm{GeV}$ and a finite width of $\Gamma=93 \mathrm{keV}$ in unpolarized twisted $e^{+} e^{-}$annihilation with the following parameters:

$E_{1}=1.8 \mathrm{GeV}, \quad E_{2}=1.338 \mathrm{GeV}, \quad\left(m_{1}, m_{2}\right)=(5 / 2,1 / 2)$, $\bar{\varkappa}_{1}=0.2 \mathrm{GeV}, \quad \bar{\varkappa}_{2}=0.1 \mathrm{GeV}, \quad \sigma_{i}=\bar{\varkappa}_{i} / 5$.

Although the energies of both incoming particles are fixed, smearing over $\varkappa_{i}$ produces a distribution in $\theta_{K}$. In Fig. 2, we show the resulting differential cross section $d \sigma_{\lambda_{V}} / d \cos \theta_{K}$ computed beyond the paraxial approximation for all three polarization states $\lambda_{V}= \pm 1,0$. With the parameter choice (17), the cross section is strongly dominated by the polarization state $\lambda_{V}=+1$, with $\mathrm{a} \approx 10 \%$ admixture of the $\lambda_{V}=0$ state and even smaller contribution from $\lambda_{V}=-1$.

Conclusions. - In summary, we proposed a novel complementary tool for doing spin physics in particle collisions. By preparing initial particles in twisted states with suitable intrinsic angular momenta and kinematics, one can access parity- and spin-dependent observables even in an unpolarized fully inclusive cross section. Fundamentally, it is possible because the initial twisted states explicitly break the left-right symmetry. We gave two illustrations of this effect: measurement of parity-violating effects in spin-0 particle production by unpolarized twisted photons and creation of polarized vector mesons in unpolarized $e^{+} e^{-}$ annihilation. A detailed study of these processes can be

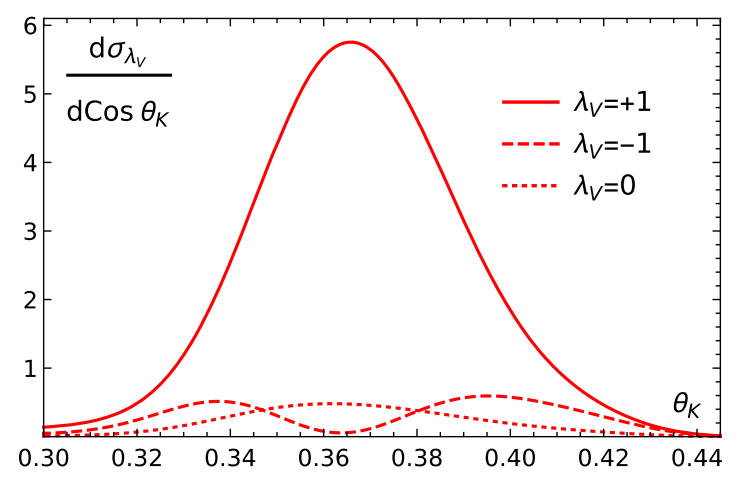

FIG. 2. Angular distribution of the $J / \psi$ meson production cross section (in arbitrary units) in unpolarized twisted $e^{+} e^{-}$annihilation with kinematic choice (17). The solid, dashed, and short dashed lines show the cross sections for $\lambda_{V}=+1,-1$, and 0 , respectively. 
found in [34]. None of these effects is possible in plane wave collisions. Combining this tool with other remarkable effects offered by twisted particle collisions discussed in [35,37-39] may result in additional insights that can be gained only in such collisions.

Understanding in which particular processes twisted particles can be advantageous over the traditional approaches is a complex research program barely started. It includes, among others, the question of what additional insights, theoretical and experimental, can be gained into the (spin) structure of the proton, once the twisted DIS is achieved.

The full exploration of these phenomena requires dedicated development of accelerator instrumentation needed to bring twisted particles into the hadronic physics realm, which will be challenging. However, many of these effects are testable even without acceleration, just with a modest experimental effort. One option is slow twisted proton and antiproton annihilation to hadrons. Also, similar effects can be observed in an atomic physics counterpart of the process we considered, e.g., in twotwisted-photon excitation of trapped atoms or ions (see Supplemental Material for details [22]). Investigation of these processes will be done in future publications. Here, we showed that nature allows us to probe her fundamental constituents and interactions in a novel way, and it is up to us to exploit this opportunity. We believe that this message and the scientific cases presented here fully justify further efforts in particle physics, beam physics, accelerator, and atomic physics communities.

I. P. I. thanks the Institute of Modern Physics, Lanzhou, China, for financial support and hospitality during his stay. I. P. I. acknowledges funding from the Portuguese Fundação para a Ciência e a Tecnologia (FCT) through the FCT Investigator Contract No. IF/00989/2014/CP1214/CT0004 and Projects No. PTDC/FIS-PAR/29436/2017, No. UIDB/ 00777/2020, and No. UIDP/00777/2020, which are partially funded through POCI, COMPETE, Quadro de Referéncia Estratégica Nacional (QREN), and the European Union. I. P. I. also acknowledges the support from National Science Center, Poland, via the project Harmonia (UMO-2015/18/M/ ST2/00518). P.M.Z. and N.K. are supported by the National Natural Science Foundation of China through Grant No. 11975320 (P.M.Z.) and No. 11875296 (N. K.). The work by A. V.P. has been partially supported by the Ministry of Education and Science of the Russian Federation: Projects No. 3.6371.2017/8.9 and No. 3.6439.2017/8.9. A. V. P. and N. K. thank the Chinese Academy of Sciences President's International Fellowship Initiative for support via Grants No. 2019PM0036 (A. V. P.) and No. 2020PM0073 (N. K.).

\footnotetext{
igor.ivanov@tecnico.ulisboa.pt

†korchagin@impcas.ac.cn

"pimikov@mail.ru
}

${ }^{\S}$ Corresponding author. zhangpm5@mail.sysu.edu.cn

[1] S. L. Olsen, Front. Phys. 10, 121 (2015).

[2] C. A. Aidala, S. D. Bass, D. Hasch, and G. K. Mallot, Rev. Mod. Phys. 85, 655 (2013).

[3] A. Accardi et al., Eur. Phys. J. A 52, 268 (2016).

[4] C. A. Aidala et al., arXiv:2002.12333.

[5] A. Bacchetta, Eur. Phys. J. A 52, 163 (2016).

[6] L. Allen, M. W. Beijersbergen, R. J. C. Spreeuw, and J. P. Woerdman, Phys. Rev. A 45, 8185 (1992).

[7] G. Molina-Terriza, J. P. Torres, and L. Torner, Nat. Phys. 3, 305 (2007).

[8] M. J. Padgett, Opt. Express 25, 11265 (2017).

[9] B. A. Knyazev and V. G. Serbo, Phys. Usp. 61, 449 (2018).

[10] K. Yu. Bliokh, Y. P. Bliokh, S. Savel'ev, and F. Nori, Phys. Rev. Lett. 99, 190404 (2007).

[11] K. Y. Bliokh et al., Phys. Rep. 690, 1 (2017).

[12] S. M. Lloyd, M. Babiker, G. Thirunavukkarasu, and J. Yuan, Rev. Mod. Phys. 89, 035004 (2017).

[13] M. Uchida and A. Tonomura, Nature (London) 464, 737 (2010).

[14] J. Verbeeck, H. Tian, and P. Schattschneider, Nature (London) 467, 301 (2010).

[15] B. J. McMorran, A. Agrawal, I. M. Anderson, A. A. Herzing, H. J. Lezec, J. J. McClelland, and J. Unguris, Science 331, 192 (2011).

[16] U. D. Jentschura and V. G. Serbo, Phys. Rev. Lett. 106, 013001 (2011).

[17] U. D. Jentschura and V. G. Serbo, Eur. Phys. J. C 71, 1571 (2011).

[18] A. J. Silenko and O. V. Teryaev, Phys. Part. Nucl. Lett. 16, 77 (2019).

[19] I. P. Ivanov, Phys. Rev. D 83, 093001 (2011).

[20] I. P. Ivanov and V. G. Serbo, Phys. Rev. A 84, 033804 (2011).

[21] D. V. Karlovets, Phys. Rev. A 86, 062102 (2012).

[22] See Supplemental Material at http://link.aps.org/ supplemental/10.1103/PhysRevLett.124.192001 for the description of twisted particles and experimental prospects, which includes Refs. [23-30].

[23] L. D. Landau and E. M. Lifshits, Quantum Mechanics, Course of Theoretical Physics (Butterworth-Heinemann, Oxford, 1991), Vol. 3.

[24] V. Serbo, I. P. Ivanov, S. Fritzsche, D. Seipt, and A. Surzhykov, Phys. Rev. A 92, 012705 (2015).

[25] V. B. Berestetskii, E. M. Lifshitz, and L. P. Pitaevskii, Quantum Electrodynamics, Course of Theoretical Physics (Pergamon Press, Oxford, 1982), Vol. 4.

[26] I. P. Ivanov, D. Seipt, A. Surzhykov, and S. Fritzsche, Europhys. Lett. 115, 41001 (2016).

[27] G. L. Kotkin, V. G. Serbo, and A. Schiller, Int. J. Mod. Phys. A 07, 4707 (1992).

[28] A. Béché, R. Van Boxem, G. Van Tendeloo, and J. Verbeeck, Nat. Phys. 10, 26 (2014).

[29] M. Krenn, J. Handsteiner, M. Fink, R. Fickler, R. Ursin, M. Malik, and A. Zeilinger, Proc. Natl. Acad. Sci. U.S.A. 113, 13648 (2016).

[30] G. M. Gallatin and B. McMorran, Phys. Rev. A 86, 012701 (2012).

[31] K. Y. Bliokh, F. J. Rodríguez-Fortuño, F. Nori, and A. V. Zayats, Nat. Photonics 9, 796 (2015). 
[32] K. Y. Bliokh, M. R. Dennis, and F. Nori, Phys. Rev. Lett. 107, 174802 (2011).

[33] D. Karlovets, Phys. Rev. A 98, 012137 (2018).

[34] I. P. Ivanov, N. Korchagin, A. Pimikov, and P. Zhang, arXiv:2002.01703.

[35] I. P. Ivanov, N. Korchagin, A. Pimikov, and P. Zhang, Phys. Rev. D 101, 016007 (2020).
[36] I. P. Ivanov, V. G. Serbo, and P. Zhang, J. Opt. 21, 114001 (2019).

[37] I. P. Ivanov, Phys. Rev. D 85, 076001 (2012).

[38] D. V. Karlovets, Europhys. Lett. 116, 31001 (2016).

[39] I. P. Ivanov, D. Seipt, A. Surzhykov, and S. Fritzsche, Phys. Rev. D 94, 076001 (2016). 